\title{
55 Die überragende Stellung der Ausdauer
}

\author{
(c) Springer-Verlag GmbH Deutschland, ein Teil von Springer Nature 2018 \\ D. Mathias, Fit und gesund von 1 bis Hundert \\ https://doi.org/10.1007/978-3-662-56307-6_55
}

Die aerobe dynamische Langzeitausdauer mit gleichmäßigem Wechselspiel von Kontraktion und Entspannung der arbeitenden Muskulatur ist fast immer Grundlage für den sportlichen Erfolg, egal ob Spitzensportler um Medaillen kämpfen oder sich Breitensportler zum Vergnügen und zur Mehrung ihrer Fitness mühen. Es ist heute sehr populär, sich beachtenswerten Ausdauerleistungen zu unterziehen, z. B. beim Laufen, Wandern, Walking, Rad fahren, Rudern, Schwimmen, Inline-Skating, Bergwandern, Skilaufen oder Tanzen.

Lebensstil, Gesundheitsstatus, soziales Umfeld und örtliche Gegebenheiten sind die wesentlichen Faktoren, die bei der Wahl der Sportart entscheiden. Die vielen Möglichkeiten der Betätigung bieten die Chance, dass alle Spaß beim Sport empfinden können.

Bewegungsarmen Menschen fällt die Aufnahme sportlicher Aktivitäten allerdings meist nicht leicht. Der Umgang mit dem eigenen Körper ist fremd geworden, oft mutet man ihm gleich zu viel $\mathrm{zu}$, der Bewegungsapparat verkündet Schmerzen, es kommt $\mathrm{zu}$ Verletzungen, schnell erlahmt das Interesse. Empfehlenswert ist daher bei dieser doch tief greifenden Lebensumstellung immer ein Beginn mit

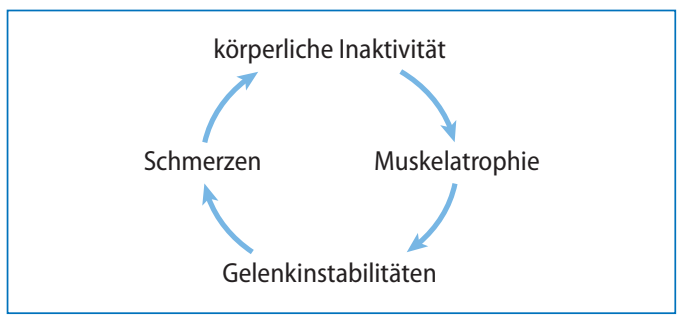

- Abb. 55.1 Auswirkung der körperlichen Inaktivität moderaten körperlichen Anstrengungen. Notwendig sind individuelle Vorgehensweisen, die Alter, Geschlecht, Erfahrung, Talent, Gewicht, Psyche und den allgemeinen Gesundheitszustand berücksichtigen. Höchste Priorität hat die Regelmäßigkeit.

Als Anhaltspunkt für geeignete Belastungsgrenzen können die von der Europäischen Atherosklerosegesellschaft vorgeschlagenen Pulsfrequenzen für »Sportentwöhnte« dienen:

- 20-29 Jahre: 115-145

- 30-39 Jahre: $110-140$

- 40-49 Jahre: $105-130$

- 50-59 Jahre: 100-125

- 60-69 Jahre: 95-115

Erst wenn ein guter Trainingszustand erreicht ist, sollte die Häufigkeit der Ausdauerübungen und danach ihr Umfang weiter gesteigert werden. Zum Erhalt der Gesundheit empfiehlt die WHO (2016) für Erwachsene wöchentlich 150 Minuten Ausdauersport. Dabei ist es egal, ob diese Anstrengungen über die Woche verteilt oder auf das Wochenende konzentriert werden (O’Donovan et al. 2017). Aber auch schon moderate körperliche Aktivitäten von täglich nur 15 Minuten erhöhen nach den Daten einer großen prospektiven Studie mit 3,4 Millionen Personenjahren deutlich die Lebenserwartung (Wen et al. 2011, 2014).

\footnotetext{
Öli-Regel: Zunächst öfter, dann auch länger und erst später intensiver trainieren. Gleichzeitige Steigerungen von Trainingsumfang und Intensität sind ausnahmsweise nur bei sehr gut trainierten Sportlern sinnvoll.
} 